\title{
1239 - Secondary Ion Mass Spectrometry for Mg Isotope Ratio Measurements: Application to Meteoritic Chondrules and Cometary Particles
}

\author{
Noriko Kita, Kohei Fukuda and Guillaume Siron \\ University of Wisconsin-Madison, Madison, Wisconsin, United States
}

Secondary Ion Mass Spectrometry (SIMS) has been applied to in-situ Mg isotope analyses of primitive meteorites and cometary particles collected by NASA Stardust Mission (comet Wild2) for understanding the early history of our Solar System [e.g., 1-2]. The ionization efficiency of $\mathrm{Mg}$ (detected $\mathrm{Mg}^{+}$ ions/sputtered $\mathrm{Mg}$ atoms) is as high as $\sim 10 \%$ by using primary $\mathrm{O}^{-}$or $\mathrm{O}_{2}^{-}$ions for WiscSIMS IMS 1280 [3], which is a significant advantage in analyzing $\mathrm{Mg}$ isotope ratios in precious small extraterrestrial samples. The WiscSIMS IMS 1280 was recently upgraded with Radiofrequency (RF) plasma ion source that improved primary beam density and stability over conventional Duoplasmatron ion source. We also developed analytical protocols of $\mathrm{Mg}$ isotope analyses; (1) to use electron multipliers (EMs) in multicollection mode and (2) to use $10^{12} \Omega$ feedback registers on Faraday cup detectors to reduce thermal noise. As a result of these new developments, we are now able to analyze $\mathrm{Mg}$ isotopes routinely from the 2-5 $\mu \mathrm{m}$ areas in meteoritic and cometary samples that were very difficult in the past.

Olivine condensates in primitive meteorites and cometary particles: Olivine in amoeboid olivine aggregates (AOAs) in primitive meteorite and nearly pure forsterite olivine particles from Wild2 are considered to have formed by condensation from hot Solar nebula gas at the birth of the Solar system. To test this hypothesis, we analyzed $\mathrm{Mg}$ isotope ratios of forsterite from AOAs in primitive $\mathrm{CO}$ chondrite and comet Wild2 particles. We also obtained minor element abundance in olivine ( $\mathrm{Ca}, \mathrm{Cr}, \mathrm{Mn}$, and $\mathrm{Fe}$ ) by SIMS with $1.5 \mu \mathrm{m}$ spot size, which is comparable to the electron microprobe (EMP) analytical volume (Fig. 1).

The results of olivine analyses show variable $\delta^{25} \mathrm{Mg}$ from $-4 \%$ o to $0 \%$ in AOAs and $-1 \%$ o to $\sim 0 \%$ in Wild 2 olivine particles. The $\mathrm{Mg}$ isotope ratios are correlated to $\mathrm{Cr}, \mathrm{Ca}$, and $\mathrm{Mn}$ concentrations of olivine, which are consistent with disequilibrium condensation of olivine from the gas of solar compositions [4]. The minor element analyses of olivine are consistent between EMP and SIMS techniques, except for FeO contents of AOA olivine, which were variable (0.1 to $1 \%)$ for EMP, but consistently lower $(\sim 0.1 \%)$ for SIMS. It seems that EMP analyses of olivine in AOAs that contain abundant small Fe-Ni metal particles would have overestimated $\mathrm{FeO}$ due to secondary fluorescence effects. Low FeO contents in AOA olivine indicated from SIMS analyses are in agreement with the condensation of olivine under the highly reducing environments predicted for Solar composition gas.

The Al-Mg chronology of chondrules: Chondrules are major components of chondritic meteorites that might have formed by transient heating processes in the protoplanetary disk. The ${ }^{26} \mathrm{Al}-{ }^{26} \mathrm{Mg}$ chronology (half-life: $7 \times 10^{5} \mathrm{y}$ ) of chondrules indicate that they postdate the formation of the oldest Ca, Al-rich inclusions (CAIs) mostly by 2-3 Ma [1]. Due to lower ${ }^{26} \mathrm{Al}$ abundance at the time of chondrule formation, resolution of a small excess ${ }^{26} \mathrm{Mg}$ (typically $\leq 2-3 \%$ ) in chondrules have been an analytical challenge. With new capabilities in WiscSIMS, we are now able to determine inferred $\left({ }^{26} \mathrm{Al} /{ }^{27} \mathrm{Al}\right)_{0}$ ratios of individual chondrules at the precisions as good as $10 \%$, equivalent to the uncertainty of $0.1 \mathrm{Ma}$ in relative ages. The analysis time per spot was reduced to 10-50 min, which were 3-8 hours in previous studies. 
In the least metamorphosed carbonaceous chondrite Acfer 094, we found two generation of chondrules that formed $\sim 1.8 \mathrm{Ma}$ and $\sim 2.4 \mathrm{Ma}$ after CAI formation, respectively [5]. The older chondrules in Acfer 094 show distinct $\mathrm{O}$ isotope and chemical signatures compared to the majority of chondrules in the same meteorite. In contrast, in ordinary chondrites, we find a limited range of ages from 1.8 Ma to 2.2 Ma [6], which are significantly different from previous studies that suggested formation ages from 1.5 Ma to 3 Ma after CAIs. Short-formation interval for ordinary chondrite chondrules are consistent with their formation in highly dense disk regions [7]. More analyses on chondrules are under progress and may help us to understand dynamical evolution of the protoplanetary disk where many different types of chondrules had formed.

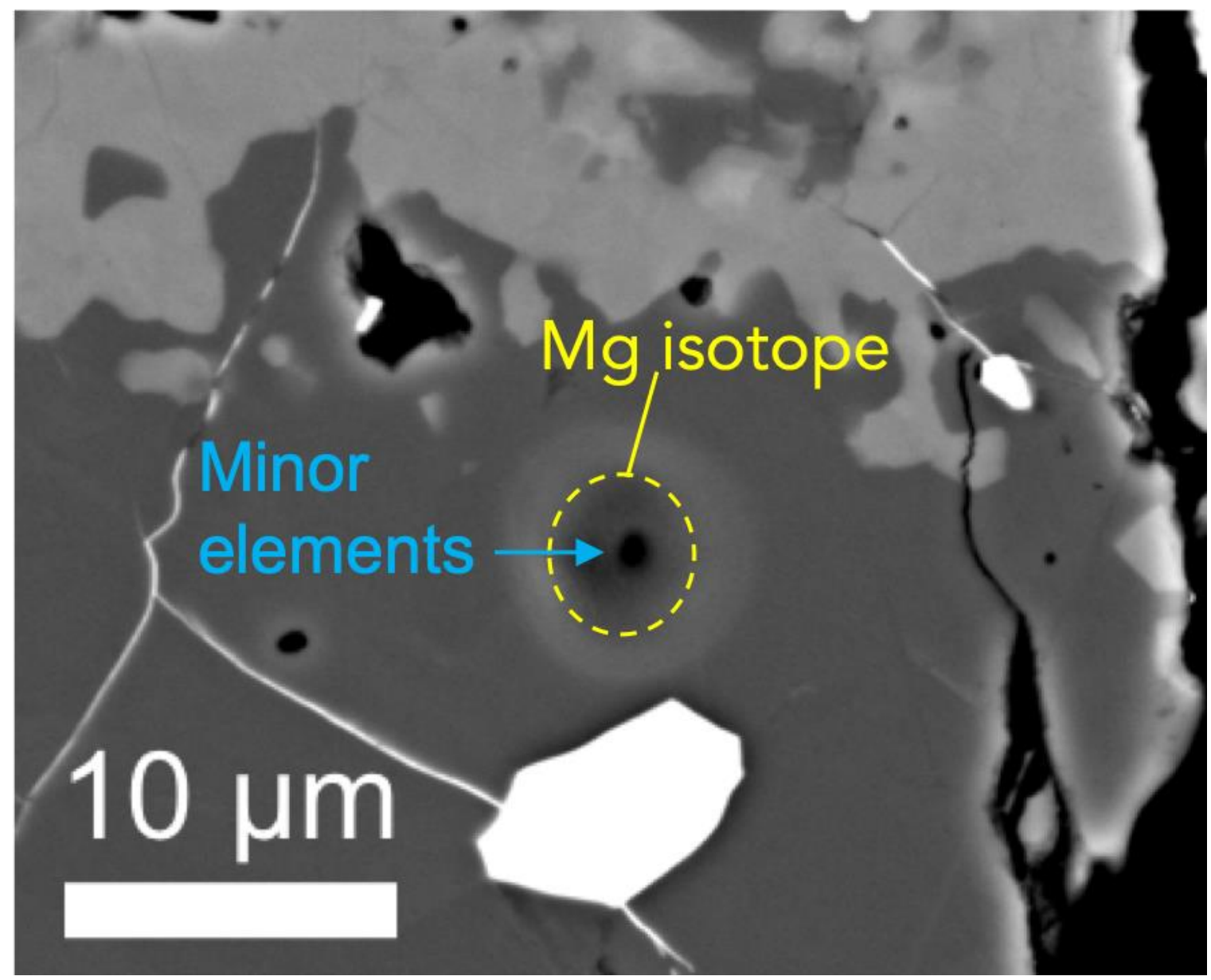

Figure 1. SIMS spots for $\mathrm{Mg}$ isotope $(5 \times 4 \mu \mathrm{m})$ and minor element $(1.5 \mu \mathrm{m})$ for olivine in AOA. Bright grain below SIMS spot is FeNi metal. 


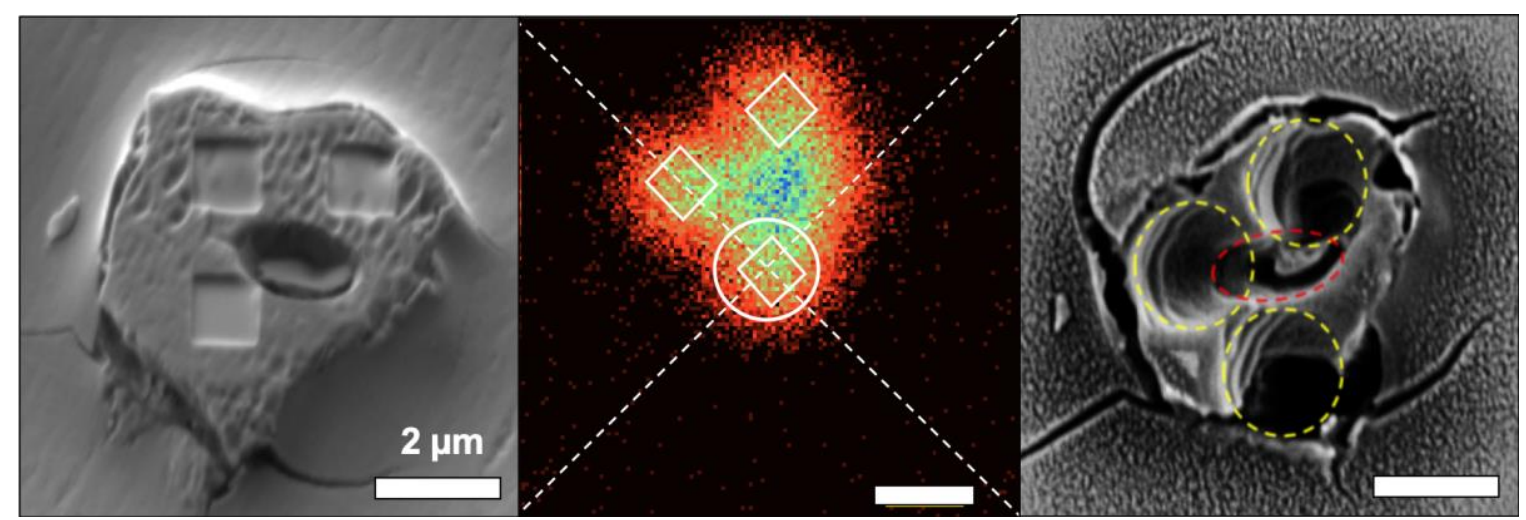

Figure 2. $\mathrm{Mg}$ isotope analyses of comet Wild2 particle (Stardust Mission). Left: The SEM image of the particle (T77/F50) that shows a previous SIMS analysis spot (using Cs primary beam) for O isotope analysis. New FIB marks were made, in which surface carbon coating was removed by $1 \mu \mathrm{m}$ squares and less than $100 \mathrm{~nm}$ deep. Middle: The FIB mark was identified by secondary $\mathrm{Mg}+$ ion image before the $\mathrm{Mg}$ isotope analysis. Positions of FIB marks and previous SIMS spot are shown as reference. Right: The SEM image of the same particle after SIMS analysis.

\section{References}

[1] NT Kita and Ushikubo, Meteoritics \& Planetary Science 47 (2012) p.1108-1119.

[2] D Nakashima et al., Earth Planetary Science Letter 410 (2015) p. 54-61.

[3] NT Kita et al., Lunar and Planetary Science Conference 48 (2017) Abstract \#1754.

[4] K Fukuda et al., Annual Meeting of the Meteoritical Society, Sapporo (2019) Abstract \# 6206.

[5] AT Hertwig et al. Goechimica et Cosmochimica Acta 253 (2019) p. 111-126.

[6] G Siron et al., Lunar and Planetary Science Conference 51 (2020) Abstract \#1574.

[7] CMO’D Alexander et al., Science 320 (2008) p. 1617-1619. 